\title{
CURRENT ANNEALINGS AND MAGNETOIMPEDANCE IN Co-Fe AMORPHOUS RIBBONS
}

\section{J. González, L. Domínguez, J.M. Blanco, P. Aragoneses}

Departmento de Fisica de Materiales, Universidad del Pais Vasco

P.O. Box 1072, 20080 San Sebastian, Spain

\section{AND R. VALENZUela}

Instituto de Investigaciones en Materiales,

National University of Mexico, P.O. Box 70-360, 04510 Mexico, Mexico

Amorphous ribbons of composition ( $\left.\mathrm{Co}_{0.95} \mathrm{Fe}_{0.05}\right)_{100-x} \mathrm{Si}_{0.4 x} \mathrm{~B}_{0.6 x}$, with $x=25$ and 30 were prepared by the melt-spinning technique. Current annealings at $600 \mathrm{~mA}$ were performed for annealing times between $15 \mathrm{~s}$ and $60 \mathrm{~min}$. The magnetoimpedance response was investigated by submitting the ribbons to ac currents at frequencies in the $10-200 \mathrm{kHz}$ range, with amplitudes between 1 and $15 \mathrm{~mA}$ (rms), and measuring their voltage response. The observed results show that a transverse anisotropy is induced by the thermal treatments, which can be evaluated by means of the magnetoimpedance effect. Due to its lower Curie temperature, the $x=30$ ribbons exhibited lower sensibility to the current annealings.

PACS numbers: $75.50 . \mathrm{Kj}, 75.60 . \mathrm{Nt}$

\section{Introduction}

Thermal annealings in amorphous ferromagnetic materials lead to an extremely wide variety of changes of magnetic domain structure and magnetic properties. Among the most important and sensitive parameters are the magnetic anisotropy and the saturation magnetostriction constants, since magnetization processes critically depend on these two basic quantities. Due to its shape, soft ferromagnetic ribbons tend naturally to have most magnetic domains in an in-plane, axial orientation. However, materials with low, negative magnetostriction constant (which can be obtained by varying the $\mathrm{Co} / \mathrm{Fe}$ ratio) offer the possibility to induce magnetic domains in a transverse geometry by means of thermal treatments such as current annealing, stress annealing, or current-stress annealing.

A novel magnetic phenomenon has recently received attention by both its basic and technological interests, the magnetoimpedance (MI) effect $[1,2]$. This phenomenon involves the impedance response of a magnetic material submitted 
to an ac current of high frequency $(1 \mathrm{kHz}-1 \mathrm{MHz})$ and low amplitude $\left(i_{\mathrm{rms}}<40 \mathrm{~mA}\right)$. When the material is additionally placed in a dc magnetic field, the impedance of the system shows a substantial decrease (about $40 \%$ of its initial value [3]). Since the field produced by the ac current has a transverse geometry, the interaction of this field is stronger with magnetic domain walls with a transverse orientation.

In this paper, MI phenomena is used to investigate transverse domains as a result of current annealings. The obtained results show that there is effectively a direct correlation between MI and the induced anisotropy.

\section{Experimental procedure}

Amorphous ribbons in the composition $\left(\mathrm{Co}_{0.95} \mathrm{Fe}_{0.05}\right)_{100-x} \mathrm{Si}_{0.4 x} \mathrm{~B}_{0.6 x}$, with $x=25$ and 30 , were prepared by the melt-spinning technique in argon atmosphere. The cross-section of the sample was $0.5 \mathrm{~mm}$ wide and $0.25 \mathrm{~mm}$ thick. Current annealings were performed at an annealing current of $600 \mathrm{~mA}$, for times between $15 \mathrm{~s}$ to $60 \mathrm{~min}$. The current annealing was carried out in air atmosphere. MI effect was measured by submitting the ribbons to ac currents in the 1-15 mA (rms) range, and frequencies in the $10 \mathrm{kHz}-200 \mathrm{kHz}$ range. The total impedance, $Z$, was measured with a voltmeter, by carefully controlling the current amplitude flowing through the ribbon. The dc magnetic field (up to $15 \mathrm{Oe}$ ) was applied by means of a Helmholtz coil system.

\section{Experimental results}

Both compositions showed the giant magnetoimpedance (GMI) effect in the as-cast condition; Fig. 1 shows the effects of the dc applied field, $H_{z}$, on the total impedance, $Z$, for $x=30$, for several frequencies. For all the frequencies, $Z$ shows the highest value at $H_{z}=0$, and as the latter increases, a strong decrease results. The effects are larger for high frequencies.

Current annealings, however, led to virtually no change in the voltage response of ribbons of this composition. Current annealings in ribbons of composition $x=25$ showed a variety of effects, as exhibited by MI. The variations in impedance as a function of applied field, for several measuring frequencies, appear

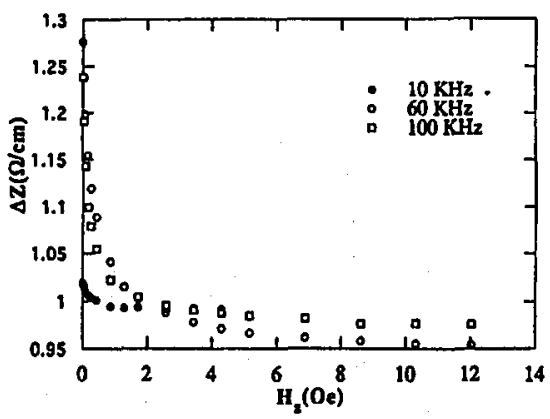

Fig. 1. Variation in total impedance, $Z$, as a function of axially applied field, $H_{z}$, for $x=30$, for several ac current frequencies. 


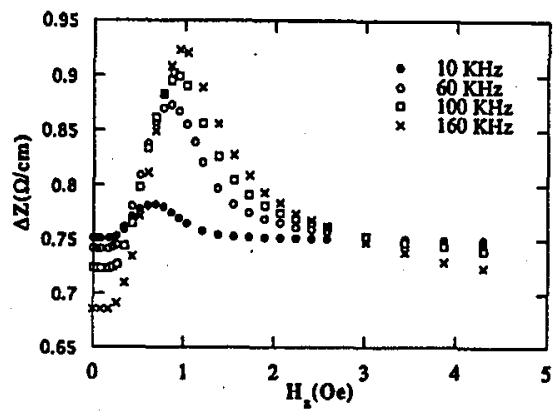

Fig. 2. Behavior of total impedance as a function of applied field for $x=25$ sample, current-annealed at $600 \mathrm{~mA}$ for $5 \mathrm{~min}$, at several frequencies.

TABLE

Annealing times, $t_{\mathrm{ann}}$ (at an annealing current of $600 \mathrm{~mA}$ ), applied field for the maximum $Z$, and $Z$ increase at the maximum (at $f=160 \mathrm{kHz}$ ).

\begin{tabular}{c|c|c}
\hline \hline$t_{\text {ann }}[\mathrm{min}]$ & $H_{\max }[\mathrm{Oe}]$ & $\Delta Z[\mathrm{~W}]$ \\
\hline $15 \mathrm{~s}$ & 0.2 & 0.3 \\
1.0 & 1.0 & 0.8 \\
2.5 & 0.5 & 0.6 \\
5.0 & 1.2 & 1.7 \\
30 & 17 & 1.7 \\
60 & 20 & 1.7
\end{tabular}

in Fig. 2, for the case of a current annealing of $600 \mathrm{~mA}$ during $5 \mathrm{~min}$. Instead of a monotonous decrease as $H_{z}$ increases, the impedance goes through a maximum. followed by a decrease in some sort similar to those observed in Fig. 1. Again, the increase in measuring frequency has an amplifying effect, since the highest frequency $(160 \mathrm{kHz})$ leads to the largest effects. The field for the maximum $Z$ increases slightly with frequency as well. The current anneal time resulted in an increase in both in the applied field for the maximum impedance, as well as in the $Z$ value. Table summarizes the dependence of the field showing the maximum $Z$, as well as the increase in $Z$, for all the annealing times.

\section{Discussion}

Since the ac current flowing through the ribbons generates an inhomogeneous transverse field, and the resulting impedance depends strongly on the coupling of this field with transverse domains, the GMI effect can be used to measure the fraction of tranverse domains in the sample. Both compositions showed the GMI effect in the as-cast state; in this condition, therefore, there exists a non-negligible amount of transverse domains. The fact that current annealings showed no change in the GMI behavior of ribbons of composition $x=30$ can be easily explained 
by recalling that current annealings have two simultaneous effects: the heating of the ribbon and the application of a strong transverse magnetic field. For the used current amplitude, the stationary-state temperature [4] of ribbons is about $340^{\circ} \mathrm{C}$. Since the Curie temperature of $x=30$ alloys is around $200^{\circ} \mathrm{C}$, it turns out that only the heating effect exists for these samples. For the $x=25$ samples, in contrast, both the heating and the applied field effects count, because $T_{\mathrm{c}}$ is as high as $400^{\circ} \mathrm{C}$.

Current annealings on $x=\mathbf{2 5}$ samples lead to the presence of an applied field needed to reach the maximum in the impedance value, Fig. 2. This field increases as the annealing time increases. Also, a kind of propagation field, i.e., a field needed to initiate domain wall displacement (indicated by the constant value of $Z$ at low fields) is observed.
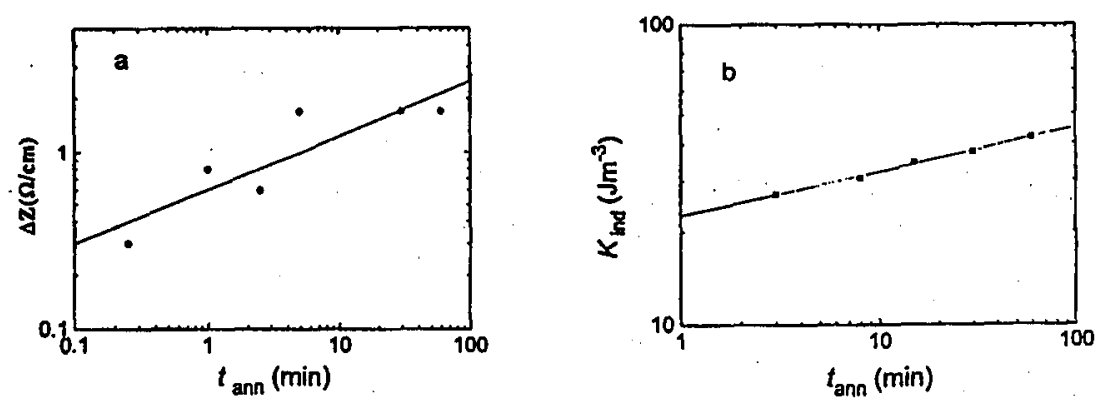

Fig. 3. (a) Difference in total impedance, $\Delta Z$, as a function of annealing time; (b) induced anisotropy as a function of $t_{\mathrm{ann}}$ for $x=25$ sample, current annealed at $600 \mathrm{~mA}$ for $5 \mathrm{~min}$. Plot (b) was adapted from [4].

In order to determine the origin of variations in GMI effect observed in the $x=25$ alloys, we compare the effects of current annealings in induced anisotropy, $K_{\text {ind }}$, and magnetostriction constant, $\lambda$, as reported in Ref. [4], with the effects on the field where the maximum in $Z$ occurs, as well as the observed $\Delta Z$ (Table), Fig. 3. All the plots are in $\log -\log$ scales to show the kinetic nature of the phenomena. The results of a simple, apparent linear fitting are also shown. Both $K_{\text {ind }}$ and $\lambda$ exhibited less dispersion than our experimental results; however, the fitting parameter is higher than 0.92 in the latter.

From Fig. 3 it appears that the induced anisotropy is mainly responsible for the observed phenomena. Our conclusion is therefore that GMI can be enhanced in Co-Fe ribbons by producing a transverse anisotropy; however, this induced anisotropy involves some domain wall pinning.

\section{References}

[1] R.S. Beach, A.E. Berkowitz, J. Appl. Phys. 76, 6209 (1994).

[2] L.V. Panina, K. Mohri, Appl. Phys. Lett. 65, 1189 (1994).

[3] K.V. Rao, F.B. Humphrey, J.L. Costa-Kramer, J. Appl. Phys. 76, 6204 (1994).

[4] M. Vazquez, J. González, A. Hernando, J. Magn. Magn. Mater. 53, 323 (1986). 